\title{
Technè
}

La science au service de l'histoire de l'art et de la préservation des biens culturels

$42 \mid 2015$

Science et conservation

\section{Nettoyage des filés d'argent ternis dans le domaine textile : comparaison du gel d'agarose et de l'éponge dite PVA comme supports poreux pour une électrolyse locale}

Cleaning tarnished silver threads in textiles: comparison between agarose gel and the PVA sponge as porous supports in local electrolysis

Julie Guerrier et Emmanuelle Pons

\section{OpenEdition}

Journals

Édition électronique

URL : http://journals.openedition.org/techne/7221

DOI : 10.4000/techne.7221

ISSN : 2534-5168

Éditeur

C2RMF

Édition imprimée

Date de publication : 1 décembre 2015

Pagination : 109-114

ISBN : 978-2-7118-6249-8

ISSN : 1254-7867

\section{Référence électronique}

Julie Guerrier et Emmanuelle Pons, « Nettoyage des filés d'argent ternis dans le domaine textile comparaison du gel d'agarose et de l'éponge dite PVA comme supports poreux pour une électrolyse locale », Technè [En ligne], 42 | 2015, mis en ligne le 01 décembre 2015, consulté le 11 mars 2021 URL : http://journals.openedition.org/techne/7221 ; DOI : https://doi.org/10.4000/techne.7221

\section{(c) (1) $\odot$}

La revue Technè. La science au service de l'histoire de l'art et de la préservation des biens culturels est mise à disposition selon les termes de la Licence Creative Commons Attribution - Pas d'Utilisation Commerciale - Pas de Modification 4.0 International. 
Julie Guerrier

Emmanuelle Pons

\section{Nettoyage des filés d'argent ternis dans le domaine textile : comparaison du gel d'agarose et de l'éponge dite PVA comme supports poreux pour une électrolyse locale}

Cleaning tarnished silver threads in textiles: comparison between agarose gel and the PVA sponge as porous supports in local electrolysis

Résumé. La conservation-restauration du textile associé à des filés d'argent ou d'argent doré ternis reste une problématique actuelle. Un traitement électrochimique permet la réduction des produits de corrosion de l'argent. Jusque-là, les modalités de ce traitement prévoient une immersion totale ou partielle de l'objet, ce qui n'est que rarement envisageable pour le textile historique. Une alternative intéressante serait de développer un stylo électrolytique adapté au textile. Dans cette étude, menée dans le cadre de l'année de mémoire de fin d'étude à l'Institut national du patrimoine ${ }^{1}$, nous proposons ainsi d'utiliser l'électrolyte avec un support poreux, afin de pouvoir traiter les filés d'argent ternis par électrolyse locale tout en gardant l'humidification sous contrôle. Deux supports poreux ont été testés, le gel d'agarose préparé à partir de la solution électrolytique, et l'éponge dite PVA. Le gel d'agarose s'avère le plus satisfaisant, car il permet un meilleur contrôle de l'humidification et laisse moins de résidus d'électrolyte, comme l'ont confirmé les analyses EDS au microscope électronique à balayage et les analyses chimiques (ICP-AES et chromatographie ionique). Un prototype de stylo à gel électrolytique a été conçu pour pallier les problèmes de manipulation rencontrés sur les essais sur éprouvettes.

Mots-clés. Filés d'argent, ternissement, électrolyse locale, textile, tissu, soie, gel d'agarose, PVA, PVFM.
Abstract. The conservation and restoration of textile with silver or gilded silver thread that have tarnished remains problematic. Reduction of the corrosion products can be obtained by an electrochemical treatment. Until now, this treatment involved soaking the object either entirely or partially in an electrolyte, a method seldom conceivable for historical textile. An interesting alternative would be to develop an electrolytic pencil adapted to textile. In this study, conducted as part of a final year dissertation at the Institut National du Patrimoine, we therefore suggested using a porous support in the electrolytic process, so as to treat the tarnished silver threads by local electrolysis, while keeping the humidification under control. Two porous supports were tested: agarose gel, prepared from the electrolytic solution, and the sponge known as PVA. Agarose gel proved to be more satisfactory, for it allowed to control the humidification more effectively and left less electrolytic residue, as was confirmed by EDS analysis on the SEM (scanning electron microscope) and chemical analysis (ICP-AES and ion chromatography). A prototype of the electrolytic gel pencil was designed to resolve handling problems met during trials involving test tubes.

Keywords. Silver threads, tarnishing, local electrolysis, textile, fabric, silk, agarose gel, PVA, PVFM.

\section{Introduction}

L'ornementation du textile à l'aide de métal est une pratique courante attestée dès l'âge du bronze ${ }^{2}$. L'or et l'argent sont des métaux de choix qui, une fois réduits à l'état de trait ou de lame extrêmement souples, peuvent être filés en les enroulant autour d'une âme textile ${ }^{3}$. Les filés ainsi obtenus peuvent ensuite être brodés, tissés, tricotés, etc. Ils apportent richesse et éclat, qualités largement exploitées par les artisans lors de leur mise en œuvre sur le textile ${ }^{4}$. L'argent plus dur et moins onéreux a souvent été préféré. Employé pur ou doré, ce métal peut toutefois poser des problèmes de corrosion. En effet, l'exposition des objets à une atmosphère contenant des polluants soufrés et chlorés ou encore leur manipulation à main nue conduit à la formation de sulfures et chlorures d'argent ${ }^{5}$.
Cette corrosion du métal argent se traduit par une perte totale d'éclat ainsi qu'une modification de la couleur de la couche de corrosion allant du jaune au bleu pour des épaisseurs croissantes, et pour un même ordre d'interférence, voire au noir si l'exposition est prolongée (formation d'une couche épaisse).

Le nettoyage de l'argent ainsi corrodé est une problématique ancienne de la conservation-restauration textile pour laquelle aucune solution satisfaisante n'a été trouvée. En effet, la méthode employée doit prendre en compte les particularités du métal qui est de faible épaisseur (inférieure au millimètre) et étroitement lié au textile sans possibilité de dissociation. Elle doit donc être compatible avec les deux matériaux, excluant de fait les méthodes mécaniques et chimiques employées jusque-là et considérées comme trop agressives

Julie Guerrier, conservateur-restaurateur du patrimoine textile (j64guerrier@gmail.com). Emmanuelle Pons, ingénieur-chercheur EDF R\&D, Laboratoire Valectra (emmanuelle.pons@edf.fr). 
pour le textile ${ }^{6}$. Restait donc l'exploration de la méthode électrochimique déjà utilisée depuis de nombreuses années en restauration du métal ${ }^{7}$.

La couche de corrosion de l'argent, généralement composée de sulfure et de chlorure d'argent, peut effectivement être éliminée par réduction électrochimique ${ }^{8}$. Pour ce faire, l'objet à traiter est relié à une alimentation stabilisée et immergé avec une contre-électrode dans une solution électrolytique. L'objet est ensuite écarté de son état d'équilibre vers le potentiel de réduction du sulfure d'argent, plus négatif que celui du chlorure d'argent, afin de réduire les deux.

Cependant, l'immersion d'un objet textile ayant un décor métallique est rarement envisageable à cause, par exemple, des risques de variation dimensionnelle de l'objet pouvant conduire à sa déformation, voire à la rupture des fibres ou bien au dégorgement des teintures. Il était donc nécessaire d'adapter la méthode afin de limiter l'humidification durant le traitement et de maîtriser les résidus d'électrolyte sur l'objet après traitement. Le contrôle de l'humidification peut passer par l'emploi d'un support poreux ayant une bonne capacité de rétention des liquides. Ce support doit également présenter une bonne souplesse afin d'épouser la surface accidentée des décors et ne pas laisser de résidus.

Deux supports ont retenu notre attention : le gel d'agar et l'éponge dite PVA. L'agar offre la possibilité de fabriquer des gels que l'on peut mouler ou découper, capables de retenir des liquides et qui ne laissent pas de résidus visibles ${ }^{9}$, même lorsqu'ils sont appliqués sur des matériaux poreux ${ }^{10}$. Ce polymère issu d'algues marines est composé de deux polysaccharides différents : l'agarose et l'agaropectine. Cette dernière contenant des sulfates ${ }^{11}$ et n'étant pas nécessaire au processus de gélification, le choix s'est porté sur l'emploi de l'agarose seul.

L'éponge en PVFM (poly(formal de vinyle)), appelée couramment éponge PVA dans le commerce, est constituée d'alcool polyvinylique que l'on fait ensuite réagir avec du formaldéhyde. Grâce à son réseau tridimensionnel de pores continus ouverts de l'ordre de la centaine de micromètre de diamètre et à une porosité très élevée, cette éponge souple et douce présente un risque d'accroche nul et un très fort pouvoir hydrophile associé à une bonne capacité de rétention des liquides.

\section{Matériaux et méthodes}

\section{Éprouvettes}

Les essais préliminaires ne pouvant être effectués sur des objets du patrimoine, des éprouvettes ont été réalisées. Le choix des matériaux, l'argent et la soie, reflète le cas le plus courant rencontré dans le textile historique.

Les filés d'argent 990 non protégés par un vernis ${ }^{12}$ ont été préalablement dégraissés à l'éthanol à 99,8 \% par immersion durant 3 minutes dans deux bains successifs. Ils ont

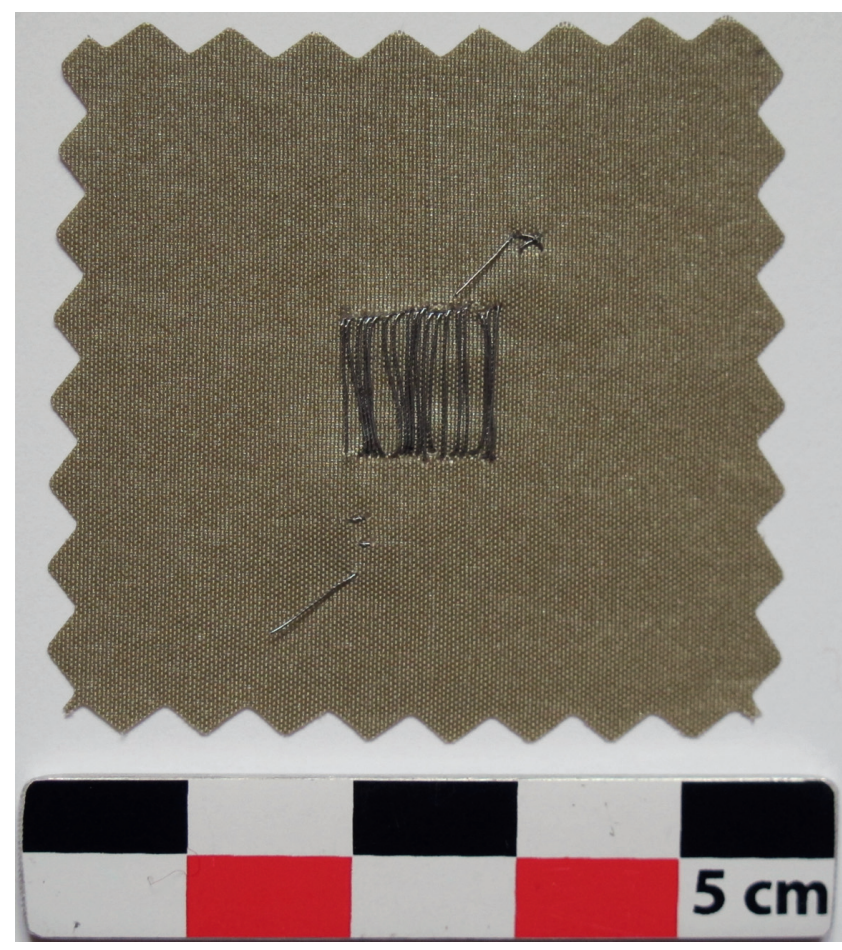

Fig. 1. Éprouvette de soie brodée de filés d'argent terni. (C) Julie Guerrier.

ensuite été suspendus sous une cloche de verre avec un bécher contenant une solution de sulfure d'ammonium ( $4 \mathrm{ml}$ de sulfure d'ammonium à $20 \%$ et $20 \mathrm{ml}$ d'eau déminéralisée $\mathrm{e}^{13}$ ) pendant 8 heures afin de les ternir. À leur sortie de cloche, les filés sont gris mat. Le tissu est un pongé de soie $\mathrm{n}^{\circ} 16$ teint avec le colorant Olive SG (Huntsman ${ }^{\circledR}$ ) afin de suivre facilement le front d'humidification causé par le traitement. Les filés d'argent ternis ont ensuite été brodés au centre de l'éprouvette en soie teinte sur une surface de $1 \mathrm{~cm}^{2}$ (fig. 1).

\section{Électrolyte et supports poreux : choix et préparation}

La soie ancienne est compatible avec l'eau déminéralisée ${ }^{14}$. Le nitrate de sodium ne modifiant pas le pH de l'eau déminéralisée, des études antérieures ont permis de sélectionner une solution de ce sel à $0,1 \mathrm{M}$ comme électrolyte.

Le premier support poreux de l'électrolyte est un gel d'agarose à $2 \%$ (2 g pour $100 \mathrm{ml}$ d'eau) réalisé à l'aide de la solution d'électrolyte ${ }^{15}$. La concentration du gel a été établie de façon à conjuguer souplesse et maîtrise de l'humidification ${ }^{16}$.

Le second support poreux est une éponge utilisée pour le nettoyage des salles blanches de classe $5^{17}$ appelée « clean room sponge $\left(\right.$ Aion $\left.^{\circledR}\right)$. Bien que référencée AION PVA Sponge sur le site du fournisseur, cette éponge non teintée est en réalité en PVFM (poly (formal de vinyle)) ${ }^{18}$. Elle est hydratée avec la solution de nitrate de sodium à $150 \%$ de sa masse ; en dessous, l'électrolyse est difficile, voire impossible ${ }^{19}$. 


\section{Techniques d'analyse}

Les éprouvettes ont été photographiées après traitement afin de pouvoir mettre en parallèle la taille des auréoles d'humidité formées et les résultats des analyses EDS et chimiques.

La présence d'éventuels résidus de l'électrolyte (sodium) est détectée par analyse EDS au microscope électronique à balayage environnemental $(\mathrm{ESEM})^{20}$. Les analyses chimiques ont été effectuées par spectrométrie d'émission optique à couplage plasma induit (ICP-AES) ${ }^{21}$ et chromatographie ionique $^{22}$. La première technique utilise un plasma généré par un couplage inductif comme source d'excitation. Elle permet ainsi de détecter les photons et de mettre en évidence les éléments traces à des teneurs allant jusqu'à 100, voire $10 \mathrm{ppb}$. La deuxième technique permet de réaliser des analyses qualitatives (par séparation des espèces présentes) et quantitatives des espèces ioniques présentes dans une solution.

\section{Protocole}

\section{Essais réalisés}

Les dimensions réduites de la zone à traiter empêchent l'utilisation d'une électrode de référence pour tous les essais. La tension aux bornes de l'alimentation stabilisée a donc été préréglée lors d'un essai préalable sur une première éprouvette, en utilisant une électrode de référence au sulfate mercureux (ESS) et dont le potentiel est de $+0,66 \mathrm{~V}$ par rapport à l'électrode normale à hydrogène ${ }^{23}$. Ceci afin d'obtenir une polarisation cathodique des filés à -1,4 V/ESS (la réduction de l'argent étant observée expérimentalement entre -1,25 et $-1,4 \mathrm{~V} / \mathrm{ESS})$. La tension correspondante est de 2,5 V. Les essais suivants ont été effectués sans électrode de référence et en conservant ce réglage de l'alimentation. La durée de la polarisation a été fixée à une minute.

Le montage de la cellule d'électrolyse est réalisé de la façon suivante : un fil de platine est glissé sous les filés brodés de l'éprouvette, qui est recouverte d'une feuille de polyester (Melinex ${ }^{\circledR}$ ) évidée au niveau de la broderie. Les filés sont

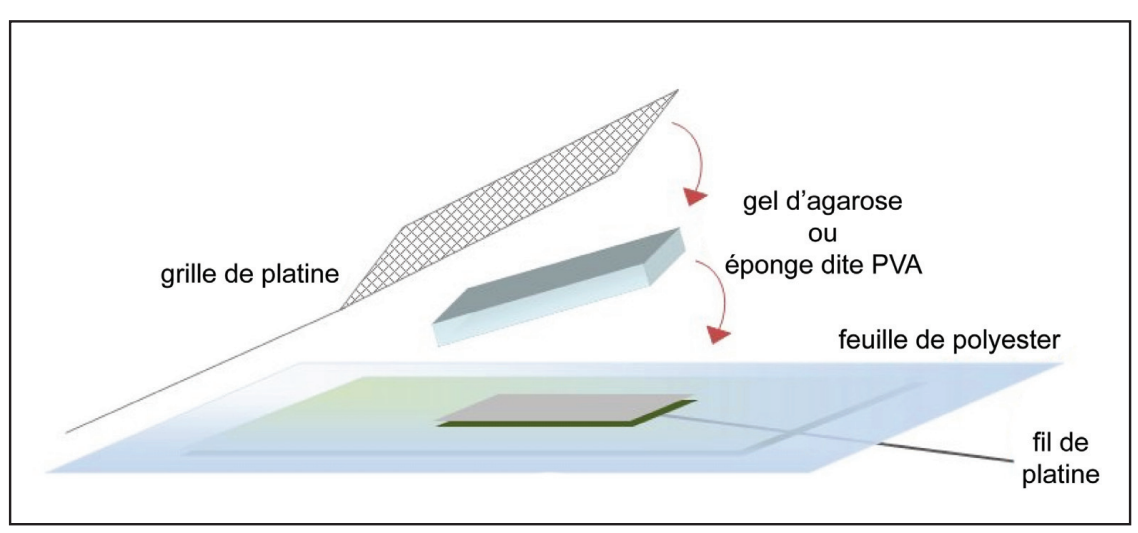

Fig. 2. Schéma de la cellule d'électrolyse. @ Julie Guerrier. alors recouverts d'un pavé de gel d'agarose préparé avec une solution de nitrate de sodium à $0,1 \mathrm{M}$ ou d'un pavé d'éponge dite PVA hydratée à $150 \%$ avec cette même solution, sur lequel on applique une grille de platine. Afin de maintenir l'ensemble en contact, une plaque de verre surmonte le tout en faisant office de poids (fig. 2).

Dans l'éventualité de résidus d'électrolyte après traitement, nous avons testé l'efficacité d'un rinçage local à l'aide de l'éponge dite PVA en l'hydratant à $50 \%$ de sa masse avec de l'eau déminéralisée. Cette quantité d'eau est nécessaire pour humidifier l'éprouvette lorsque l'éponge est pressée, tout en étant rapidement réabsorbée par l'éponge en relâchant la pression ${ }^{24}$. L'éponge est changée à chaque rinçage afin de ne pas risquer une contamination des éprouvettes et elle est de même dimension que la broderie ${ }^{25}$.

Nous avons testé pour chaque support poreux (gel et éponge) trois séries de trois éprouvettes chacune : une série non rincée après électrolyse (SR), une série rincée une fois (RE1) et une série rincée deux fois (RE2).

\section{Recherche des résidus}

En raison de la faible concentration de l'électrolyte choisi, nous n'avons pas pu détecter les éventuels résidus d'électrolyte sur les éprouvettes après traitement en mode observation au microscope électronique à balayage environnemental ${ }^{26}$. La détection de la présence des résidus est donc réalisée grâce à l'analyse EDS d'un champ d'analyse de $1 \mathrm{~mm}^{2}$ répété trois fois sur des zones prédéterminées des éprouvettes. Il ne s'agit pas d'effectuer une analyse quantitative mais seulement d'obtenir une indication quant à la présence ou l'absence de sodium sur les éprouvettes après traitement. Une éprouvette par série a été ainsi examinée en plus de l'éprouvette témoin, soit sept éprouvettes en tout.

Des analyses chimiques ont également été réalisées afin de quantifier les résidus en solution après lixiviation des éprouvettes (analyse du sodium par ICP-AES et des nitrates par chromatographie ionique). Dans ce but, un témoin, une éprouvette par série sans rinçage et une avec un rinçage (autres que celles analysées par EDS) ont été lixiviés dans $25 \mathrm{ml}$ d'eau déminéralisée ultra pure pendant 48 heures à $21^{\circ} \mathrm{C}$ en statique puis sous agitation.

\section{Résultats et discussion}

Les premiers résultats obtenus par l'analyse EDS permettent de distinguer l'éponge du gel d'agarose. En effet, toutes les éprouvettes traitées avec l'éponge, y compris celles rincées, ont suffisamment de résidus de sodium pour que cela soit détecté lors de l'analyse EDS. Il en va de même pour celle traitée 
avec le gel de $\mathrm{NaNO}_{3}$ qui n’a pas été rincée. Les éprouvettes traitées avec le gel d'agarose puis rincées ne présentent aucun résidu de sodium détectable ${ }^{27}$ comme le montre le tableau 1.

\section{Tableau 1. Résultats des analyses EDS du sodium à l'ESEM}

\begin{tabular}{|l|l|}
\hline Éprouvettes & Présence de Na \\
\hline PVA_SR_NaNO $\mathrm{Na}_{3-} 0,1 \mathrm{M}$ & $>\mathrm{LD}$ \\
\hline PVA_RE1_ $\mathrm{NaNO}_{3 \_} 0,1 \mathrm{M}$ & $>\mathrm{LD}$ \\
\hline PVA_RE2_ $\mathrm{NaNO}_{3 \_} 0,1 \mathrm{M}$ & $>\mathrm{LD}$ \\
\hline GEL_SR_NaNO $\mathrm{NaN}_{3} 0,1 \mathrm{M}$ & $>\mathrm{LD}$ \\
\hline GEL_RE1_ $\mathrm{NaNO}_{3 \_} 0,1 \mathrm{M}$ & $<\mathrm{LD}$ \\
\hline GEL_RE2_ $\mathrm{NaNO}_{3 \_} 0,1 \mathrm{M}$ & $<\mathrm{LD}$ \\
\hline TÉMOIN & $<\mathrm{LD}$ \\
\hline
\end{tabular}

Les résultats sont cohérents avec la taille des auréoles d'humidité qui se sont formées au cours du traitement. En effet, les éprouvettes dont les auréoles ont été les plus importantes sont celles de la série traitée avec l'éponge. À l'inverse, les éprouvettes des séries réalisées avec le gel d'agarose ne présentaient que de très petites auréoles ${ }^{28}$.

Le gel permet donc un meilleur contrôle de l'humidification des éprouvettes et une limitation du dépôt de résidus d'électrolytes.

Les résultats des analyses chimiques du sodium et des ions nitrates sur deux éprouvettes traitées avec l'éponge et deux éprouvettes traitées avec le gel d'agarose sont donnés dans le tableau 2.

Tableau 2. Résultats d'analyse du sodium par ICP-AES et des ions nitrates par chromatographie ionique

\begin{tabular}{|c|c|c|}
\hline & \multicolumn{2}{|c|}{ Concentration $(\mu \mathrm{g} / \mathrm{L})$} \\
\hline Éprouvettes & $\mathrm{Na}$ & $\mathrm{NO}_{3}$ \\
\hline PVA_SR_NaNO ${ }_{3-} 0,1 \mathrm{M}$ & 5511 & 16000 \\
\hline PVA_RE1_NaNO ${ }_{3-} 0,1 \mathrm{M}$ & 2929 & 6900 \\
\hline $\mathrm{GEL}_{-} \mathrm{SR} \_\mathrm{NaNO}_{3-0,1 \mathrm{M}}$ & 1079 & 1743 \\
\hline GEL_RE1_NaNO ${ }_{3-} 0,1 \mathrm{M}$ & 953 & 1459 \\
\hline TÉMOIN & 228 & 135 \\
\hline
\end{tabular}

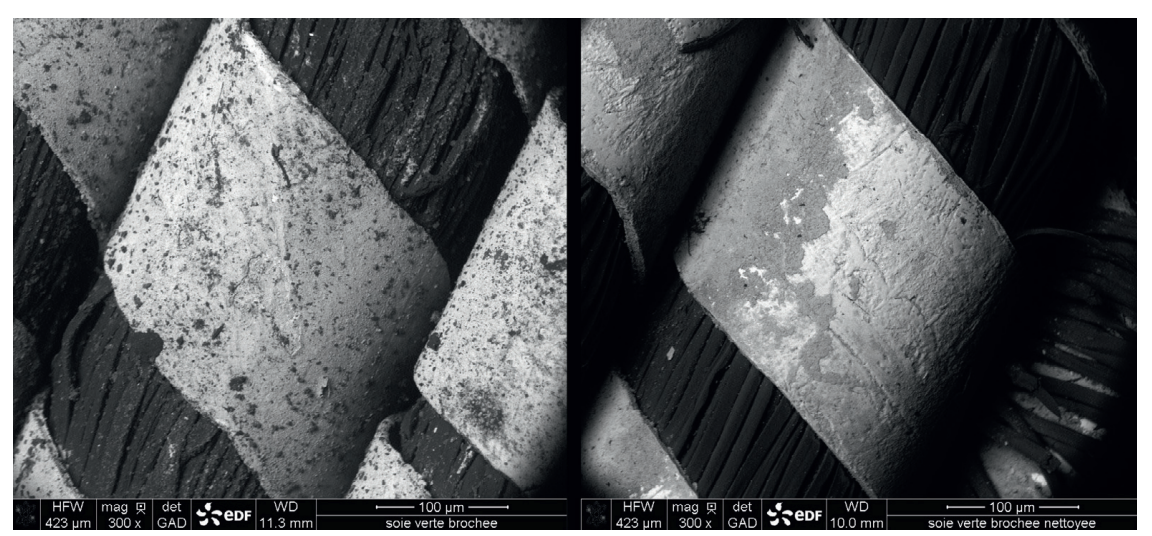

Après traitement, l'éclat est retrouvé, même lorsque le nettoyage est incomplet (fig. 4). Cependant, la cellule d'électrolyse employée durant l'expérience est difficile à utiliser sur un objet du patrimoine. En effet, le gel d'agarose

Fig. 3. Exemple de filés d'argent doré avant (à gauche) et après nettoyage (à droite) par électrolyse. Image ESEM en mode électrons rétrodiffusés. La couche d'or dégagée apparaît en blanc sur l'image de droite. () EDF. 


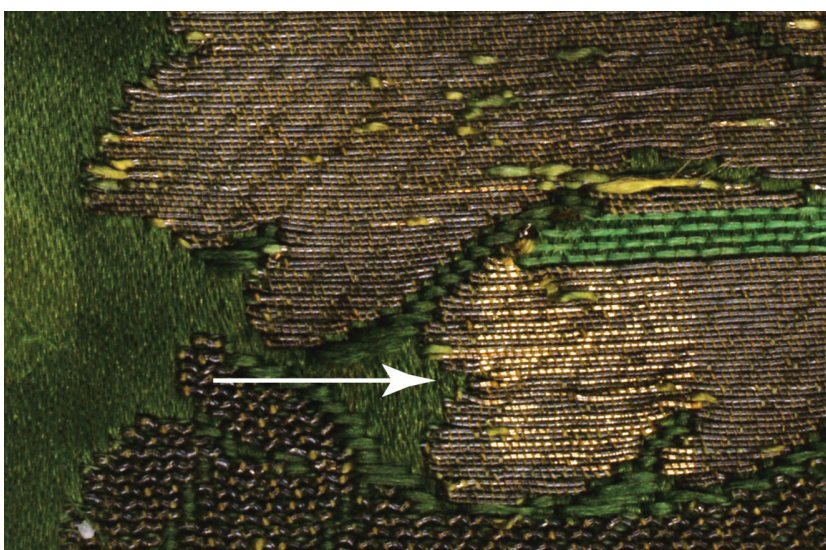

Fig. 4. Essai de nettoyage de filés d'argent doré brochés sur soie avec un carré de gel d'agarose préparé avec la solution de $\mathrm{NaNO}_{3}$ à $0,1 \mathrm{M}$. La flèche indique la zone nettoyée. (C) Julie Guerrier.

est complexe à manipuler, le cache en Melinex ${ }^{\circledR}$ n'empêche pas la diffusion de l'électrolyte par capillarité dans le tissu et le contact entre tous les éléments est délicat à assurer par une personne seule.

Afin d'améliorer ces différents points, nous avons pensé réunir le gel d'agarose et le connecteur dans le tube en polypropylène d'une seringue, afin de pouvoir manipuler ces deux éléments ensemble à la manière d'un stylo. L'autre main est ainsi libre pour placer sur l'objet le fil de platine ou plus simplement une épingle en inox afin d'établir le contact électrique (fig. 5).

Le prototype fabriqué a été réalisé avec des matériaux peu coûteux : un emporte-pièce tube et son poussoir en inox (1) utilisé en cuisine, une seringue (2) de diamètre identique à l'emporte-pièce, un morceau de mousse de polyéthylène (3), une cosse (4) et un câble de cuivre (5) terminé par une fiche banane (6). La cosse est brasée à l'argent à l'extrémité du poussoir afin de connecter le câble la reliant à l'alimentation stabilisée. La mousse de polyéthylène est découpée à l'aide de l'emporte-pièce et fendue à la verticale pour bloquer le poussoir dans le tube de la seringue La pastille de gel

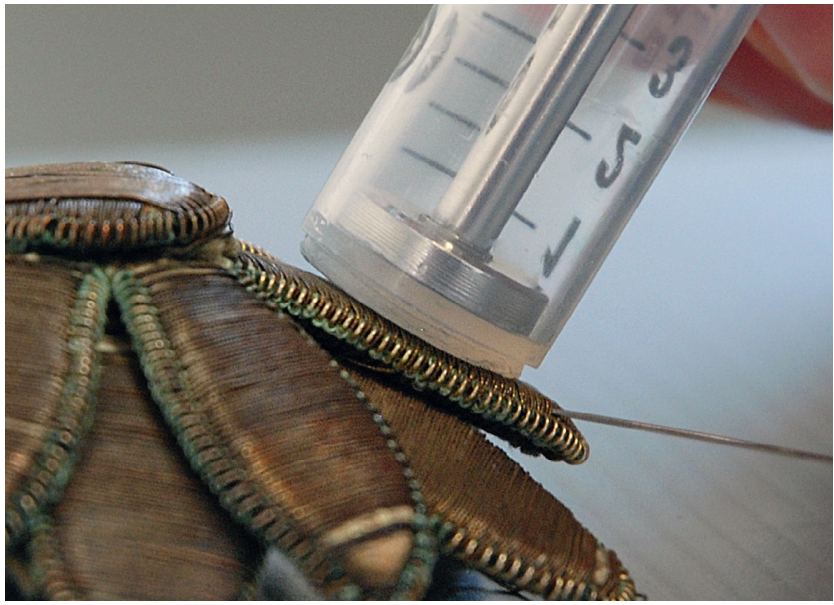

Fig. 5. Exemple d'utilisation du prototype sur un élément de passementerie ancien. (C) Julie Guerrier.

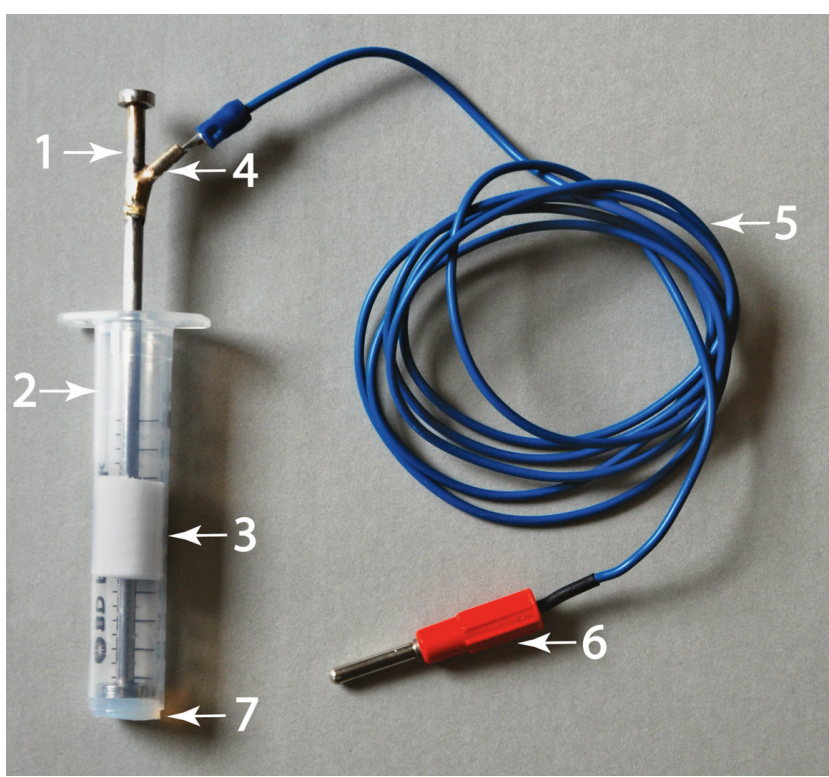

Fig. 6. Prototype de stylo à gel électrolytique. (c) Julie Guerrier.

d'agarose (7) est découpée avec l'emporte-pièce et vient se loger dans la seringue contre le poussoir en inox (fig. 6).

\section{Conclusion et perspectives}

Le gel d'agarose s'avère être le meilleur support pour le nitrate de sodium dans le cas d'une électrolyse locale sans immersion dans le domaine textile. En effet, il permet un meilleur contrôle de l'humidification et, comme le montrent les résultats des analyses EDS et chimiques, laisse moins de résidus d'électrolyte que l'éponge dite PVA, tout en permettant un nettoyage de l'argent terni par électrolyse. Les problèmes de manipulation rencontrés ont été résolus de manière satisfaisante par le prototype. Celui-ci, associé à l'emploi du cyclododécane ${ }^{31}$ pour isoler le tissu autour du métal à traiter, pourrait constituer une solution intéressante pour nettoyer l'argent terni par électrolyse, tout en ayant un bon contrôle de l'humidification et des résidus d'électrolyte.

D'autres paramètres mériteraient d'être explorés comme le mélange de gels différents pour améliorer la souplesse et donc le contact avec l'objet durant le traitement. Il pourrait être intéressant de vérifier les effets de la polarisation sur les teintures textiles pour prendre en compte les cas où l'âme des filés serait teinte. L'étude pourrait également être étendue aux filés ayant une âme en fibres cellulosiques.

\section{Remerciements}

Nous tenons à remercier le centre de microscopie, le laboratoire de chimie et le laboratoire polymères et composites d'EDF Lab Les Renardières, Dominique de Reyer du LRMH, l'INP, ainsi que toutes les personnes ayant participé à ce projet. 


\section{Notes}

1. Ce mémoire porte sur l'étude et la restauration de chaussures des XVII ${ }^{\mathrm{e}}$ et XVIII ${ }^{\mathrm{e}}$ siècles brodées de filés métalliques (directeur de mémoire : Patricia Dal-Prà).

2. Gleba, 2008, p. 61

3. L'âme textile est généralement composée d'un faisceau de fibres de soie blanche ou teinte en jaune pour les filés d'argent ou d'argent doré.

4. Note de la rédaction : voir article de A. Villa et A. Bos, «Éléments de technique et de vocabulaire sur la broderie d'or à l'époque moderne, autour d'un manteau de l'ordre du Saint-Esprit conservé au musée du Louvre ", dans Technè, n 41, p. 55-64.

5. Bouquet, 1993, p.463 ; Franey, 1985, p. 133

6. Tímár-Balázsy, Eastop, 1998, p. 245.

7. Degrigny, 2010, p. 353.

8. Costa, 2003, p. 287.

9. Warda, 2007, p. 275

10. Les gels rigides d'agar et agarose sont déjà utilisés pour nettoyer de nombreux supports comme le papier, le plâtre, la terre cuite, le textile...

11. Le sulfate peut éventuellement occasionner une corrosion du cuivre qui entre souvent dans la composition de l'argent utilisé dans le domaine textile.

12. Le titre de l'argent est de 990 millièmes, ce qui correspond à la quantité d'argent dans l'alliage. Fournisseur Carlhian.

13. Marcos, Degrigny, 1992, p. 29

14. Tímár-Balázsy, Eastop, 1998, p. 43 ; Stemann-Petersen, Taarnskov, 2006, p. 288.

15. La préparation du gel suit les étapes suivantes : la solution de nitrate de sodium est mise à chauffer sous agitation magnétique dans un bécher au bain-marie. Quand elle atteint $40^{\circ} \mathrm{C}$, l'agarose en poudre est versé dans le bécher. La préparation est chauffée jusqu'à atteindre $90{ }^{\circ} \mathrm{C}$ et maintenue à cette température quelques minutes. Lorsque la préparation est claire, elle est versée dans un récipient. Le gel doit refroidir à température ambiante afin de permettre une réticulation correcte. Ce mode de préparation du gel a été dicté par le constat que lui seul nous garantit la conservation de la concentration de l'électrolyte à $0,1 \mathrm{M}$.

16. Guerrier, 2014, p. 143

17. La norme NF EN ISO 14644-1 (1999), intitulée « Salles propres et environnements maîtrisés apparentés, Partie 1 : Classification de la propreté de l'air », définit la propreté particulaire et identifie 9 classes (de 1 à 9, la classe ISO 1 étant la plus propre). La classe ISO 5 correspond à une concentration maximale admissible de 100000 particules de taille égale ou supérieure à $0,1 \mu \mathrm{m}$ par $\mathrm{m}^{3}$ d'air.

18. Degrigny, 2014, p. 72. Donnée vérifiée par une analyse en calorimétrie différentielle à balayage (appareillage utilisé DSC 214 Polyma NETZSCH) : température de transition vitreuse déterminée à $107,5^{\circ} \mathrm{C}$ à la vitesse de $2 \mathrm{~K} / \mathrm{min}$, valeur proche de la température indiquée dans la bibliographie : $105^{\circ} \mathrm{C}$ (cf. Lampman, 2003, p.137).
19. Guerrier, 2014, p. 141.

20. ESEM FEG Quanta 600.

21. Thermo iCAP 6500.

22. Metrohm IC 820.

23. XR200, Radiometer Analytical.

24. Guerrier, 2014, p. 145.

25. Guerrier, 2014, p. 145.

26. Guerrier, 2014, p. 138.

27. La limite de détection est à $0,1 \%$ massique. Mais l'analyse EDS ne peut pas être considérée dans notre cas comme une analyse quantitative. Les pourcentages massiques indiqués par l'appareillage sont uniquement à prendre pour une indication de présence de sodium (quantité supérieure à la limite de détection) ou d'absence (quantité de sodium inférieure à la limite de détection). En effet, l'analyse en pourcentage massique n'est valable que pour un échantillon massif, homogène et dont la surface est polie.

28. Guerrier, 2014, p. 153.

29. Fragment provenant du fond d'étude de Dominique de Reyer, LRMH.

30. Guerrier, 2014, p. 158 ; Vacquié, 1994, p. 31 ; Degrigny, 1996, p. 172 ; Degrigny, 2014, p. 64.

31. Le cyclododécane est souvent employé en restauration textile pour isoler temporairement une partie sensible durant un traitement aqueux, ce produit a notamment la propriété de rendre une surface hydrophobe et de se sublimer à température ambiante.

\section{Bibliographie}

Bouquet S., Bodin C., Fiaud C., 1993 , «Influence relative des composés soufrés et chlorés sur le ternissement de l'argent en milieu atmosphérique ", compte rendu de l'Académie des Sciences, Paris, t. 316, Série II, p. $459-464$

Costa V., 2003, « L'électrolyse appliquée au traitement des œuvres en argent du patrimoine ", Bulletin, $\mathrm{n}^{\circ}$ 30, IRPA, Bruxelles, p. 287-297.

Degrigny C., Wéry M., Vescoli V., Blengino M., 1996, «Altération et nettoyage de pièces en argent doré », Studies in Conservation, vol. 41, n 3 , p. 170-178.

Degrigny C., 2010, "Use of electrochemical techniques for the conservation of metal artifacts: a review", Journal of solid state electrochemistry, vol. 14, n ${ }^{\circ} 3$, p. 353 361.

Franey J. P., Kammlott G. W., Graedel T. E., 1985, "The corrosion of silver by atmospheric sulfurous gases", Corrosion Science, vol. 25, p. 133-143.

Gleba M., 2008, "Auratae vestes: gold textiles in the ancient Mediterranean" dans Pupureae Vestes. II Symposium Internacional sobre textiles y tintes des Mediterráneo en el mundo antiguo, Valencia, p. 61-77.

Lampman S., 2003, Characterization and failure analysis of plastics, ASM International.
Stemann-Petersen K., Taarnskov B., 2006, "Electrolytic cleaning of silver and gilt silver threads in silk textiles possibilities and limitation", ZKK Journal for Art Technology and Conservation, $\mathrm{n}^{\circ}$ 2, p. 278288.

Tímár-Balázsy Á., Eastop D., 1998, Chemical principles of textile conservation, Butterworth-Heinemann, Oxford.

Warda J., Brückle I., Bezur A., Kushel D., 2007, "Analysis of Agarose, Carbopol and Laponite Gel Poultices in Paper Conservation", Journal of the American Institute for Conservation, vol. $46, \mathrm{n}^{\circ} 3$, p. 263-279.

\section{Documents inédits}

Degrigny C., 2014, Mise au point de méthodes de nettoyage électrolytique innovantes pour la restauration de chefs-d'œuvre d'orfèvrerie composites, Application au trésor de l'abbaye de Saint-Maurice d'Agaune, Rapport final, Projet Sagex n 31516, Haute École Arc, Neuchâtel.

Guerrier J., 2014, Étude et conservationrestauration de chaussures des XVII et XVIII ${ }^{e}$ siècles (Musée international de la chaussure, Romans), le nettoyage des filés d'argent ternis brodés sur soie : comparaison de gel d'agarose et de l'éponge PVA comme support poreux en vue d'une électrolyse locale sans immersion, mémoire de fin d'études, Inp, Paris.

Marcos F., Degrigny C., 1992, Mise au point du traitement de conservation d'une partie de la collection des cuivres du Musée de la musique de Paris, Rapport EDF, Groupe des Laboratoires, Valectra, RA 921155.

Vacquié C., 1994, Mise au point d'un traitement de nettoyage de surfaces en argent doré ternies - Étude sur des filés métalliques de franges de drapeaux (association métaltextile), Rapport EDF, Groupe des Laboratoires, Valectra, RB 941994.

Vacquié C., Blengino J.-M., Lacoudre N., 1995, «Traitement électrolytiques de franges de drapeau ternies par la corrosion atmosphérique ", Cahier technique, $\mathrm{n}^{\circ} 1$, ARAAFU, Paris, p. 19-26.

\section{Fournisseurs}

Filé d'argent 990 non verni sur âme de soie non teinte, Carlhian, Lyon.

Toile de soie : pongé $n^{\circ} 16$, Ponsard.

Colorant textile : Olive SG Huntsman ${ }^{\circledR}$, Grande-Bretagne.

Éponge D-3 « clean room sponge », Aion ${ }^{\circledR}$.

Agarose ADN Electran ${ }^{\circledR}$ pour électrophorèse, VWR.

Fil de platine, Heraeus.

Électrode de référence au sulfate mercureux, Radiometer Analytical.

Set Design culinaire, 5 tubes découpoirs et poussoirs, Tellier ${ }^{\circledR}$. 